\title{
A NEW $\left(\gamma_{n}, \sigma_{k}\right)$-KP HIERARCHY AND GENERALIZED DRESSING METHOD
}

\author{
YUQIN YAO \\ Department of Applied Mathematics, China Agricultural University \\ Beijing 100083, P. R. China \\ yyqinw@126.com \\ YEHUI HUANG \\ School of Mathematics and Physics, North China Electric Power University \\ Beijing 102206, P. R. China \\ huangyh@mails.tsinghua.edu.cn \\ YUNBO ZENG \\ Department of Mathematical Science, Tsinghua University \\ Beijing 100084, P. R. China \\ yzeng@math.tsinghua.edu.cn \\ Received 20 March 2012 \\ Accepted 30 May 2012 \\ Published 31 December 2012
}

\begin{abstract}
A new $\left(\gamma_{n}, \sigma_{k}\right)$-KP hierarchy (KPH) with two new time series $\gamma_{n}$ and $\sigma_{k}$, which consists of $\gamma_{n}$-flow, $\sigma_{k}$-flow and mixed $\gamma_{n}$ and $\sigma_{k}$ evolution equations of eigenfunctions, is proposed. Two reductions and constrained flows of $\left(\gamma_{n}, \sigma_{k}\right)-\mathrm{KPH}$ are studied. The dressing method is generalized to the $\left(\gamma_{n}, \sigma_{k}\right)-\mathrm{KPH}$ and some solutions are presented.
\end{abstract}

Keywords: $\left(\gamma_{n}, \sigma_{k}\right)$-KP hierarchy; constrained flows; Lax representation; generalized dressing method.

\section{Introduction}

Generalizations of KP hierarchy $(\mathrm{KPH})$ attract a lot of interests from both physical and mathematical points of view $[2-4,7,8,15-17,19,21,22]$. One kind of generalization is the multi-component KPH [2], which contains many physical relevant nonlinear integrable systems such as Davey-Stewartson equation, two-dimensional Toda lattice and three-wave resonant integrable equations. Another kind of generalization of KP equation is the so-called KP equation with self-consistent sources (KPESCS) [15, 16]. For example, the first type and second type of KPESCS consists of KP equation with some additional terms and eigenvalue problem or time evolution equations of eigenfunctions of KP equation, respectively [5, 6 , $15-17,23]$. 
Denote the time series of KPH by $\left\{t_{n}\right\}$. Recently, we proposed an approach to construct an extended KPH (exKPH) by introducing another time series $\left\{\tau_{k}\right\}[10,13,24]$. The exKPH consists of $t_{n}$-flow of $\mathrm{KPH}, \tau_{k}$-flow and the $t_{n}$-evolution equations of eigenfunctions. To make difference, we may call the exKPH as $\left(t_{n}, \tau_{k}\right)$-KPH. The $\left(t_{n}, \tau_{k}\right)$-KPH contains the first type and second type of KPESCS. Also we developed the dressing method to solve the $\left(t_{n}, \tau_{k}\right)$ $\mathrm{KPH}$ [12]. The paper [14] generalized the $\left(t_{n}, \tau_{k}\right)-\mathrm{KPH}$ to the $\left(\tau_{n}, \tau_{k}\right)-\mathrm{KPH}$ which consists of $\tau_{n}$-flow, $\tau_{k}$-flow and the $\tau_{n}$-evolution equations of eigenfunctions and $\tau_{k}$-evolutions of eigenfunctions. However, [14] did not find the solution of $\left(\tau_{n}, \tau_{k}\right)-\mathrm{KPH}$. In contrast to one $t_{n}$-evolution equation of eigenfunctions as coupling equation in our $\left(t_{n}, \tau_{k}\right)$-KPH, there are two coupling equations: $\tau_{n}$-evolution and $\tau_{k}$-evolution equations of eigenfunctions in $\left(\tau_{n}, \tau_{k}\right)$ $\mathrm{KPH}$. Our generalized dressing method cannot be applied to the $\left(\tau_{n}, \tau_{k}\right)$-KPH due to too many coupling equations.

In this paper, we generalize the $\left(t_{n}, \tau_{k}\right)-\mathrm{KPH}$ to $\left(\gamma_{n}, \sigma_{k}\right)$-KPH by introducing two new time series $\gamma_{n}$ and $\sigma_{k}$ with two parameters $\alpha_{n}$ and $\beta_{k}$. The $\left(\gamma_{n}, \sigma_{k}\right)$-KPH consists of $\gamma_{n}$-flow, $\sigma_{k}$-flow and one mixed $\gamma_{n}$ and $\sigma_{k}$ evolution equation of eigenfunctions. The $\left(\gamma_{n}, \sigma_{k}\right)$-KPH can be reduced to the $\mathrm{KPH}$ and $\left(t_{n}, \tau_{k}\right)-\mathrm{KPH}$, and contains first type and second type as well as mixed type of KPESCS as special cases. The constrained flows of the $\left(\gamma_{n}, \sigma_{k}\right)$-KPH can be regarded as generalization of Gelfand-Dickey hierarchy (GDH), which contains the first type, second type as well as mixed type of GDH with self-consistent sources in special cases. We also develop the dressing method to solve the $\left(\gamma_{n}, \sigma_{k}\right)-\mathrm{KPH}$. Comparing with the multi-component generalization, we generalize the KPH by means of introducing two new time series and adding eigenfunctions as components.

The paper is organized as follows: In Sec. 2 , we propose a new $\left(\gamma_{n}, \sigma_{k}\right)$-KPH. Section 3 presents the constrained flows of the $\left(\gamma_{n}, \sigma_{k}\right)$-KPH. Section 4 devotes to develop the generalized dressing method for solving the $\left(\gamma_{n}, \sigma_{k}\right)$-KPH. Section 5 presents the $N$-soliton solutions and a conclusion is given in the Sec. 6.

\section{A New $\left(\gamma_{n}, \sigma_{k}\right)$-KP Hierarchy}

\subsection{The KP hierarchy and extended KP hierarchy}

Let us first recall the construction of the $\mathrm{KPH}[2-4,7,21]$ and the exKPH $[12,13]$. It is well known that the pseudo-differential operator $L$ with potential functions $u_{i}$ is defined as

$$
L=\partial+u_{1} \partial^{-1}+u_{2} \partial^{-2}+\cdots .
$$

The KPH is given by [4]

$$
L_{t_{n}}=\left[B_{n}, L\right]
$$

where $B_{n}=L_{+}^{n}$ stands for the differential part of $L^{n}$. The compatibility of the $t_{n}$-flow and $t_{k}$-flow of (1) leads to the zero-curvature representation of $\mathrm{KPH}$

$$
B_{n, t_{k}}-B_{k, t_{n}}+\left[B_{n}, B_{k}\right]=0 .
$$

In particular, $B_{2}=\partial^{2}+u_{1}, B_{3}=\partial^{3}+3 u_{1} \partial+3\left(u_{1 x}+u_{2}\right)$ and $(2)$ by setting $t_{2}=y, t_{3}=t$ and $u_{1}=u$ yields the $\mathrm{KP}$ equation

$$
\left(4 u_{t}-12 u u_{x}-u_{x x x}\right)_{x}-3 u_{y y}=0 .
$$


Based on the observation that the squared eigenfunction symmetry constraint given by

$$
\begin{aligned}
L^{k} & =B_{k}+\sum_{i=1}^{N} q_{i} \partial^{-1} r_{i}, \\
q_{i, t_{n}} & =B_{n}\left(q_{i}\right), \quad r_{i, t_{n}}=-B_{n}^{*}\left(r_{i}\right)
\end{aligned}
$$

is compatible with $\mathrm{KPH}[1,9]$, we proposed the exKPH as follows in [13]

$$
\begin{aligned}
L_{t_{n}} & =\left[B_{n}, L\right], \\
L_{\tau_{k}} & =\left[B_{k}+\sum_{i=1}^{N} q_{i} \partial^{-1} r_{i}, L\right], \\
q_{i, t_{n}} & =B_{n}\left(q_{i}\right), \quad r_{i, t_{n}}=-B_{n}^{*}\left(r_{i}\right), \quad i=1, \ldots, N .
\end{aligned}
$$

The commutativity of (3a) and (3b) under (3c) gives rise to the following zero-curvature representation for exKPH (3)

$$
\begin{aligned}
& B_{n, \tau_{k}}-B_{k, t_{n}}+\left[B_{n}, B_{k}\right]+\left[B_{n}, \sum_{i=1}^{N} q_{i} \partial^{-1} r_{i}\right]_{+}=0 \\
& q_{i, t_{n}}=B_{n}\left(q_{i}\right), \quad r_{i, t_{n}}=-B_{n}^{*}\left(r_{i}\right), \quad i=1, \ldots, N
\end{aligned}
$$

To different (3) from (1) and the generalized KPH presented in this paper, we may denote (3) or $(4)$ by $\left(t_{n}, \tau_{k}\right)$-KPH. We developed the dressing method to solve the $\left(t_{n}, \tau_{k}\right)$-KPH and obtained its solutions in [12]. The paper [14] generalized the $\left(t_{n}, \tau_{k}\right)-\mathrm{KPH}$ to $\left(\tau_{n}, \tau_{k}\right)-\mathrm{KPH}$ as follows

$$
\begin{aligned}
& B_{n, \tau_{k}}-B_{k, \tau_{n}}+\left[B_{n}, B_{k}\right]+\left[B_{n}, \sum_{i=1}^{N} q_{i} \partial^{-1} r_{i}\right]_{+}+\left[\sum_{i=1}^{N} q_{i} \partial^{-1} r_{i}, B_{k}\right]_{+}=0 \\
& q_{i, \tau_{n}}=B_{n}\left(q_{i}\right), \quad r_{i, \tau_{n}}=-B_{n}^{*}\left(r_{i}\right), \\
& q_{i, \tau_{k}}=B_{k}\left(q_{i}\right), \quad r_{i, \tau_{k}}=-B_{k}^{*}\left(r_{i}\right), \quad i=1, \ldots, N
\end{aligned}
$$

But [14] did not find the solutions for the $\left(\tau_{n}, \tau_{k}\right)$-KPH (5). In contrast to one pair of coupling equations $(3 \mathrm{c})$ (or $(4 \mathrm{~b}))$ in $\left(t_{n}, \tau_{k}\right)-\mathrm{KPH}$, there are two pairs of coupling equations $(5 \mathrm{~b})$ and $(5 \mathrm{c})$ in $\left(\tau_{n}, \tau_{k}\right)$-KPH. In fact, the dressing method developed in our paper [12] cannot be applied to the $\left(\tau_{n}, \tau_{k}\right)$-KPH (5) since there are too many (two) coupling systems $(5 \mathrm{~b})$ and $(5 \mathrm{c})$.

\subsection{A new $\left(\gamma_{n}, \sigma_{k}\right)-K P$ hierarchy}

Stimulated by the $\left(t_{n}, \tau_{k}\right)$-KPH (3) and (4), we propose the following generalized KPH with two generalized time series $\gamma_{n}$ and $\sigma_{k}$ :

$$
L_{\gamma_{n}}=\left[B_{n}+\alpha_{n} \sum_{i=1}^{N} q_{i} \partial^{-1} r_{i}, L\right],
$$




$$
\begin{aligned}
& L_{\sigma_{k}}=\left[B_{k}+\beta_{k} \sum_{i=1}^{N} q_{i} \partial^{-1} r_{i}, L\right], \\
& \alpha_{n}\left(q_{i, \sigma_{k}}-B_{k}\left(q_{i}\right)\right)-\beta_{k}\left(q_{i, \gamma_{n}}-B_{n}\left(q_{i}\right)\right)=0, \\
& \alpha_{n}\left(r_{i, \sigma_{k}}+B_{k}^{*}\left(r_{i}\right)\right)-\beta_{k}\left(r_{i, \gamma_{n}}+B_{n}^{*}\left(r_{i}\right)\right)=0, \quad i=1,2, \ldots, N .
\end{aligned}
$$

We will prove the compatibility of (6a) and (6b) under (6c) in the following theorem. First we need the following Lemma presented in [13]

$$
\left[B_{n}, \sum_{i=1}^{N} q_{i} \partial^{-1} r_{i}\right]_{-}=\sum_{i=1}^{N} B_{n}\left(q_{i}\right) \partial^{-1} r_{i}-\sum_{i=1}^{N} q_{i} \partial^{-1} B_{n}^{*}\left(r_{i}\right) .
$$

Theorem 1. The $\gamma_{n}$-flow (6a) and $\sigma_{k}$-flow (6b) under (6c) are compatible.

Proof. Denote

$$
\begin{aligned}
& \tilde{B}_{n}=B_{n}+\alpha_{n} \sum_{i=1}^{N} q_{i} \partial^{-1} r_{i}, \\
& \tilde{B}_{k}=B_{k}+\beta_{k} \sum_{i=1}^{N} q_{i} \partial^{-1} r_{i} .
\end{aligned}
$$

In order to prove $L_{\gamma_{n}, \sigma_{k}}=L_{\sigma_{k}, \gamma_{n}}$, i.e.

$$
\left[\tilde{B}_{n, \sigma_{k}}-\tilde{B}_{k, \gamma_{n}}+\left[\tilde{B}_{n}, \tilde{B}_{k}\right], L\right]=0
$$

we only need to prove

$$
\tilde{B}_{n, \sigma_{k}}-\tilde{B}_{k, \gamma_{n}}+\left[\tilde{B}_{n}, \tilde{B}_{k}\right]=0 .
$$

For convenience, we omit $\sum$. We can find that

$$
\begin{aligned}
\tilde{B}_{n, \sigma_{k}} & =B_{n, \sigma_{k}}+\alpha_{n}\left(q \partial^{-1} r\right)_{\sigma_{k}}=\left[B_{k}+\beta_{k}\left(q \partial^{-1} r\right), L^{n}\right]_{+}+\alpha_{n}\left(q \partial^{-1} r\right)_{\sigma_{k}} \\
& =\left[B_{k}, L^{n}\right]_{+}+\beta_{k}\left[q \partial^{-1} r, L^{n}\right]_{+}+\alpha_{n} q_{\sigma_{k}} \partial^{-1} r+\alpha_{n} q \partial^{-1} r_{\sigma_{k}},
\end{aligned}
$$

and similarly,

$$
\tilde{B}_{k, \gamma_{n}}=\left[B_{n}, L^{k}\right]_{+}+\alpha_{n}\left[q \partial^{-1} r, L^{k}\right]_{+}+\beta_{k} q_{\gamma_{n}} \partial^{-1} r+\beta_{k} q \partial^{-1} r_{\gamma_{n}} .
$$

Making use of the basic Lemma (7), we have

$$
\begin{aligned}
{\left[\tilde{B}_{n}, \tilde{B}_{k}\right]=} & {\left[B_{n}, B_{k}\right]+\left[B_{n}, \beta_{k} q \partial^{-1} r\right]+\left[\alpha_{n} q \partial^{-1} r, B_{k}\right] } \\
= & {\left[L^{n}-\left(L^{n}\right)_{-}, L^{k}-\left(L^{k}\right)_{-}\right]_{+}+\beta_{k}\left[B_{n}, q \partial^{-1} r\right]_{+}+\alpha_{n}\left[q \partial^{-1} r, B_{k}\right]_{+} } \\
& +\beta_{k}\left[B_{n}, q \partial^{-1} r\right]_{-}+\alpha_{n}\left[q \partial^{-1} r, B_{k}\right]_{-} \\
= & {\left[B_{n}, L^{k}\right]_{+}+\left[L^{n}, B_{k}\right]_{+}-\left[\left(L^{n}\right)_{-},\left(L^{k}\right)_{-}\right]_{+} } \\
& +\beta_{k}\left[B_{n}, q \partial^{-1} r\right]_{+}+\alpha_{n}\left[q \partial^{-1} r, B_{k}\right]_{+} \\
& +\beta_{k} B_{n}(q) \partial^{-1} r-\beta_{k} q \partial^{-1} B_{n}^{*}(r) \\
& -\alpha_{n} B_{k}(q) \partial^{-1} r+\alpha_{n} q \partial^{-1} B_{k}^{*}(r) .
\end{aligned}
$$


Then (9), (10) and (11) under (6c) yields

$$
\begin{aligned}
\tilde{B}_{n, \sigma_{k}}-\tilde{B}_{k, \gamma_{n}}+\left[\tilde{B}_{n}, \tilde{B}_{k}\right]= & {\left[\alpha_{n}\left(q_{\sigma_{k}}-B_{k}(q)\right)-\beta_{k}\left(q_{\gamma_{n}}-B_{n}(q)\right)\right] \partial^{-1} r } \\
& +q \partial^{-1}\left[\alpha_{n}\left(r_{\sigma_{k}}+B_{k}^{*}(r)\right)-\beta_{k}\left(r_{\gamma_{n}}+B_{n}^{*}(r)\right)\right]=0 .
\end{aligned}
$$

Then the compatibility of $\gamma_{n}$-flow (6a) and $\sigma_{k}$-flow $(6 \mathrm{~b})$ under (6c) gives rise to the zero-curvature representation for (6)

$$
\begin{gathered}
\left(B_{n}+\alpha_{n} \sum_{i=1}^{N} q_{i} \partial^{-1} r_{i}\right)_{\sigma_{k}}-\left(B_{k}+\beta_{k} \sum_{i=1}^{N} q_{i} \partial^{-1} r_{i}\right)_{\gamma_{n}} \\
+\left[B_{n}+\alpha_{n} \sum_{i=1}^{N} q_{i} \partial^{-1} r_{i}, B_{k}+\beta_{k} \sum_{i=1}^{N} q_{i} \partial^{-1} r_{i}\right]=0
\end{gathered}
$$

which under (6c) can be simplified as follows. Then we have the following theorem.

Theorem 2. The commutativity of (6a) and (6b) under (6c) gives rise to the zero-curvature equation for the generalized KPH with two generalized time series

$$
\begin{aligned}
& B_{n, \sigma_{k}}-B_{k, \gamma_{n}}+\left[B_{n}, B_{k}\right]+\beta_{k}\left[B_{n}, \sum_{i=1}^{N} q_{i} \partial^{-1} r_{i}\right]_{+} \\
& \quad+\alpha_{n}\left[\sum_{i=1}^{N} q_{i} \partial^{-1} r_{i}, B_{k}\right]_{+}=0 \\
& \alpha_{n}\left(q_{i, \sigma_{k}}-B_{k}\left(q_{i}\right)\right)-\beta_{k}\left(q_{i, \gamma_{n}}-B_{n}\left(q_{i}\right)\right)=0, \\
& \alpha_{n}\left(r_{i, \sigma_{k}}+B_{k}^{*}\left(r_{i}\right)\right)-\beta_{k}\left(r_{i, \gamma_{n}}+B_{n}^{*}\left(r_{i}\right)\right)=0, \quad i=1,2, \ldots, N,
\end{aligned}
$$

with the Lax representation

$$
\psi_{\gamma_{n}}=\left(B_{n}+\alpha_{n} \sum_{i=1}^{N} q_{i} \partial^{-1} r_{i}\right)(\psi), \quad \psi_{\sigma_{k}}=\left(B_{k}+\beta_{k} \sum_{i=1}^{N} q_{i} \partial^{-1} r_{i}\right)(\psi) .
$$

We briefly call (6) and (12) as $\left(\gamma_{n}, \sigma_{k}\right)$-KPH. It is easy to see that $\left(\gamma_{n}, \sigma_{k}\right)$-KPH $(6)$ and (12) for $\alpha_{n}=\beta_{k}=0$ reduces to $\mathrm{KPH}(1)$ and (2), $\left(\gamma_{n}, \tau_{k}\right)-\mathrm{KPH}$ for $\alpha_{n}=0, \beta_{k}=1$ reduces to $\left(t_{n}, \tau_{k}\right)-\mathrm{KPH}(3)$ and $(4)$. So $\left(\gamma_{n}, \sigma_{k}\right)-\mathrm{KPH}(6)$ and (12) present a more generalized KPH which contains the $\mathrm{KPH}$ and $\left(t_{n}, \tau_{k}\right)-\mathrm{KPH}$ as the special cases.

Example 1. Let us take $n=2$ and $k=3$, and set $\gamma_{2}=y, \sigma_{3}=t, u_{1}=u$. Then Eqs. (12) becomes

$$
\begin{aligned}
B_{2, t} & -B_{3, y}+\left[B_{2}, B_{3}\right]+\beta_{3}\left[B_{2}, \sum_{i=1}^{N} q_{i} \partial^{-1} r_{i}\right]_{+} \\
& +\alpha_{2}\left[\sum_{i=1}^{N} q_{i} \partial^{-1} r_{i}, B_{3}\right]_{+}=0
\end{aligned}
$$




$$
\begin{aligned}
& \alpha_{2}\left(q_{i, t}-B_{3}\left(q_{i}\right)\right)-\beta_{3}\left(q_{i, y}-B_{2}\left(q_{i}\right)\right)=0, \\
& \alpha_{2}\left(r_{i, t}+B_{3}^{*}\left(r_{i}\right)\right)-\beta_{3}\left(r_{i, y}+B_{2}^{*}\left(r_{i}\right)\right)=0, \quad i=1,2, \ldots, N,
\end{aligned}
$$

which gives the following nonlinear equation

$$
\begin{aligned}
& 4 u_{t}-3 \partial^{-1} u_{y y}-12 u u_{x}-u_{x x x}-3 \alpha_{2} \sum_{i=1}^{N}\left(q_{i} r_{i}\right)_{y}+4 \beta_{3} \sum_{i=1}^{N}\left(q_{i} r_{i}\right)_{x} \\
& +3 \alpha_{2} \sum_{i=1}^{N}\left(q_{i} r_{i, x x}-q_{i, x x} r_{i}\right)=0, \\
& \alpha_{2}\left(q_{i, t}-q_{i, x x x}-3 u q_{i, x}-\frac{3}{2} q_{i} \partial^{-1} u_{y}-\frac{3}{2} q_{i} u_{x}-\frac{3}{2} q_{i} \sum_{j=1}^{N} q_{j} r_{j}\right) \\
& -\beta_{3}\left(q_{i, y}-q_{i, x x}-2 u q_{i}\right)=0, \\
& \alpha_{2}\left(r_{i, t}-r_{i, x x x}-3 u r_{i, x}+\frac{3}{2} r_{i} \partial^{-1} u_{y}-\frac{3}{2} r_{i} u_{x}+\frac{3}{2} r_{i} \sum_{j=1}^{N} q_{j} r_{j}\right) \\
& -\beta_{3}\left(r_{i, y}+r_{i, x x}+2 u r_{i}\right)=0, \quad i=1,2, \ldots, N,
\end{aligned}
$$

with the Lax representation as follows

$$
\begin{aligned}
& \psi_{y}=\left(\partial^{2}+2 u+\alpha_{2} \sum_{i=1}^{N} q_{i} \partial^{-1} r_{i}\right)(\psi) \\
& \psi_{t}=\left(\partial^{3}+3 u \partial+\frac{3}{2} \partial^{-1} u_{y}+\frac{3}{2} u_{x}+\frac{3}{2} \beta_{3} \sum_{i=1}^{N} q_{i} \partial^{-1} r_{i}\right)(\psi) .
\end{aligned}
$$

Specially, when take $\alpha_{2}=\beta_{3}=0 ; \alpha_{2}=0, \beta_{3}=1 ; \alpha_{2}=1, \beta_{3}=0$ and $\alpha_{2}=1$, $\beta_{3}=1$, respectively, (15) and (16) reduces to the KP equation [4], the first type of KPESCS $[15,16,23]$, the second type of KPESCS $[6,13,15]$ and the mixed type of KPESCS [6] and their Lax representations, respectively.

\section{Reduction}

Consider the constraint given by

$$
L^{k}=B_{k}+\beta_{k} \sum_{i=1}^{N} q_{i} \partial^{-1} r_{i} .
$$

Then (6b) yields

$$
\begin{gathered}
\left(L^{k}\right)_{\sigma_{k}}=\left[B_{k}+\beta_{k} \sum_{i=1}^{N} q_{i} \partial^{-1} r_{i}, L^{k}\right]=0, \\
B_{k, \sigma_{k}}=\left(L_{\sigma_{k}}^{k}\right)_{+}=0 \\
\left(\sum_{i=1}^{N} q_{i} \partial^{-1} r_{i}\right)_{\sigma_{k}}=\left(L_{\sigma_{k}}^{k}\right)_{-}=0
\end{gathered}
$$


which imply that $L, B_{k}, q_{i}$ and $r_{i}$ under (17) are independent of $\sigma_{k}$. Subsequently, $q_{i, \sigma_{k}}$ and $r_{i, \sigma_{k}}$ in (6c) should be replaced by $\lambda_{i} q_{i}$ and $-\lambda_{i} r_{i}$ as in the case of constrained flow of KP $[1,9]$, namely (6c) under the constraint (17) should be replaced by

$$
\begin{aligned}
\alpha_{n}\left(\lambda_{i} q_{i}-B_{k}\left(q_{i}\right)\right)-\beta_{k}\left(q_{i, \gamma_{n}}-B_{n}\left(q_{i}\right)\right) & =0, \\
\alpha_{n}\left(-\lambda_{i} r_{i}+B_{k}^{*}\left(r_{i}\right)\right)-\beta_{k}\left(r_{i, \gamma_{n}}+B_{n}^{*}\left(r_{i}\right)\right) & =0 .
\end{aligned}
$$

We will show that the constraint (17) is invariant under the $\gamma_{n}$-flow (6a) and (19). In fact, making use of (6a), (7) and (19), we have

$$
\begin{aligned}
\left(L^{k}-B_{k}\right)_{\gamma_{n}}= & \left(L_{\gamma_{n}}^{k}\right)_{-}=\left[\tilde{B}_{n}, L^{k}\right]_{-} \\
\left(\beta_{k} \sum_{i=1}^{N} q_{i} \partial^{-1} r_{i}\right)_{\gamma_{n}}= & \beta_{k} \sum_{i=1}^{N}\left(q_{i, \gamma_{n}} \partial^{-1} r_{i}+q_{i} \partial^{-1} r_{i, \gamma_{n}}\right) \\
= & \sum_{i=1}^{N}\left[\beta_{k} B_{n}\left(q_{i}\right) \partial^{-1} r_{i}+\alpha_{n}\left(\lambda_{i} q_{i}-B_{k}\left(q_{i}\right)\right) \partial^{-1} r_{i}\right. \\
& \left.\left.-\beta_{k} q_{i} \partial^{-1} B_{n}^{*}\left(r_{i}\right)+\alpha_{n} q_{i} \partial^{-1}\left(-\lambda_{i} r_{i}+B_{k}^{*}\left(r_{i}\right)\right)\right]_{-}^{N}\right]_{-}^{N} \\
= & {\left[B_{n}, \beta_{k} \sum_{i=1}^{N} q_{i} \partial^{-1} r_{i}\right]_{i=1}-\left[B_{k}, \alpha_{n} \sum^{-1} r_{i}\right]_{-} } \\
= & {\left[\tilde{B}_{n}, \beta_{k} \sum_{i=1}^{N} q_{i} \partial^{-1} r_{i}\right]_{-}\left[B_{k}, \alpha_{n} \sum_{i=1} q_{i} \partial^{-1} r_{i}\right]_{-} } \\
= & {\left[\tilde{B}_{n}, L^{k}\right]_{-}\left[\tilde{B}_{n}, B_{k}\right]_{-}-\left[B_{k}, \tilde{B}_{n}\right]_{-}+\left[B_{k}, B_{n}\right]_{-} } \\
= & {\left[\tilde{B}_{n}, L^{k}\right]_{-} }
\end{aligned}
$$

Then

$$
\left(L^{k}-B_{k}-\beta_{k} \sum_{i=1}^{N} q_{i} \partial^{-1} r_{i}\right)_{\gamma_{n}}=0 .
$$

This means that the sub-manifold determined by the $k$-constraint (17) is invariant under the $\gamma_{n}$-flow (6a) and (19).

Therefore, the constrained flow of $\left(\gamma_{n}, \sigma_{k}\right)$-KPH (6) and (12) under (17) reads

$$
\begin{aligned}
& B_{k, \gamma_{n}}+\left[B_{k}, B_{n}\right]+\beta_{k}\left[\sum_{i=1}^{N} q_{i} \partial^{-1} r_{i}, B_{n}\right]_{+}+\alpha_{n}\left[B_{k}, \sum_{i=1}^{N} q_{i} \partial^{-1} r_{i}\right]_{+}=0, \\
& \alpha_{n}\left(\lambda_{i} q_{i}-B_{k}\left(q_{i}\right)\right)-\beta_{k}\left(q_{i, \gamma_{n}}-B_{n}\left(q_{i}\right)\right)=0, \\
& \alpha_{n}\left(-\lambda_{i} r_{i}+B_{k}^{*}\left(r_{i}\right)\right)-\beta_{k}\left(r_{i, \gamma_{n}}+B_{n}^{*}\left(r_{i}\right)\right)=0, \quad i=1,2, \ldots, N .
\end{aligned}
$$


with

$$
B_{n}=\left(B_{k}+\beta_{k} \sum_{i=1}^{N} q_{i} \partial^{-1} r_{i}\right)_{+}^{\frac{n}{k}} .
$$

The system (20) can be regarded as the generalized GDH. When $\alpha_{n}=\beta_{k}=0$, (20) reduces to the GDH. When $\alpha_{n}=1, \beta_{k}=0,(20)$ is just the first type of GDH with selfconsistent sources. When $\alpha_{n}=0, \beta_{k}=1,(20)$ represent the second type of GDH with self-consistent sources.

Example 2. When $k=2, n=3, \gamma_{3}=t, u_{1}=u$, (20) gives

$$
\begin{aligned}
& u_{t}-\frac{1}{4} u_{x x x}-3 u u_{x}+\alpha_{3} \sum_{i=1}^{N}\left(q_{i} r_{i}\right)_{x}+\frac{3}{4} \beta_{2} \sum_{i=1}^{N}\left(q_{i} r_{i, x x}-q_{i, x x} r_{i}\right)=0, \\
& -\beta_{2}\left(q_{i, t}-q_{i, x x x}-3 u q_{i, x}-\frac{3}{2} u_{x} q_{i}-\frac{3}{2} q_{i} \sum_{j=1}^{N} q_{j} r_{j}\right) \\
& +\alpha_{3}\left(\lambda_{i} q_{i}-q_{i, x x}-2 u q_{i}\right)=0, \\
& \beta_{2}\left(r_{i, t}-r_{i, x x x}-3 u r_{i, x}-\frac{3}{2} u_{x} r_{i}+\frac{3}{2} r_{i} \sum_{j=1}^{N} q_{j} r_{j}\right) \\
& -\alpha_{3}\left(-\lambda_{i} r_{i}+r_{i, x x}+2 u r_{i}\right)=0,
\end{aligned}
$$

Which is just the mixed type of $\mathrm{KdV}$ equation with self-consistent sources. Equation (21) with $\alpha_{3}=1, \beta_{2}=0$ gives the first type of $\mathrm{KdV}$ equation with sources [11, 18]. Equation (21) with $\alpha_{3}=0, \beta_{2}=1$ gives the second type of $\mathrm{KdV}$ equation with sources [13].

Example 3. When $k=3, n=2$ and $\gamma_{2}=t, u_{1}=u,(20)$ gives rise to the mixed type of Boussinesq equation with self-consistent sources

$$
\begin{aligned}
& \frac{1}{3} u_{x x x x}+2\left(u^{2}\right)_{x x}+u_{t t} \\
& \quad+\sum_{i=1}^{N}\left[-\frac{4}{3} \beta_{3}\left(q_{i} r_{i}\right)_{x x}+\alpha_{2}\left(q_{i} r_{i}\right)_{x t}+\alpha_{2}\left(q_{i, x x} r_{i}-q_{i} r_{i, x x}\right)_{x}\right]=0 \\
& \alpha_{2}\left[\lambda_{i} q_{i}-q_{i, x x x}-3 u q_{i, x}-q_{i}\left(\frac{3}{2} \partial^{-1} u_{y}+\frac{3}{2} u_{x}+\frac{3}{2} \sum_{i=1}^{N} q_{j} r_{j}\right)\right] \\
& \quad-\beta_{3}\left(q_{i, t}-q_{i, x x}-2 u q_{i}\right)=0, \\
& \alpha_{2}\left[\begin{array}{l}
\left.-\lambda_{i} r_{i}-r_{i, x x x}-3 u r_{i, x}+r_{i}\left(\frac{3}{2} \partial^{-1} u_{y}-\frac{3}{2} u_{x}+\frac{3}{2} \sum_{i=1}^{N} q_{j} r_{j}\right)\right] \\
\quad-\beta_{3}\left(r_{i, t}-r_{i, x x}-2 u r_{i}\right)=0, \quad i=1,2, \ldots, N .
\end{array}\right.
\end{aligned}
$$


Equation (22) with $\alpha_{2}=1, \beta_{3}=0$ gives the first type of Boussinesq equation with sources. Equation (21) with $\alpha_{2}=0, \beta_{3}=1$ gives the second type of Boussinesq equation with sources [13].

\section{Dressing Approach for $\left(\gamma_{n}, \sigma_{k}\right)-\mathrm{KPH}$}

Inspired by $[4,20]$, we consider the generalized dressing approach for $\left(\gamma_{n}, \sigma_{k}\right)$-KPH. Assume that operator $L$ of $\left(\gamma_{n}, \sigma_{k}\right)-\mathrm{KPH}$ can be written as a dressing form

$$
\begin{gathered}
L=W \partial W^{-1} \\
W=1+w_{1} \partial^{-1}+w_{2} \partial^{-2}+\cdots
\end{gathered}
$$

Proposition 1. If $W$ defined by (24) satisfies

$$
\begin{aligned}
& W_{\gamma_{n}}=-L_{-}^{n} W+\alpha_{n} \sum_{i=1}^{N} q_{i} \partial^{-1} r_{i} W \\
& W_{\sigma_{k}}=-L_{-}^{k} W+\beta_{k} \sum_{i=1}^{N} q_{i} \partial^{-1} r_{i} W
\end{aligned}
$$

then $L$ satisfies (6a) and (6b).

Proof. Based on (23) and (25a), we have

$$
\begin{aligned}
L_{\gamma_{n}} & =W_{\gamma_{n}} \partial W-W \partial W^{-1} W_{\gamma_{n}} W^{-1} \\
& =\left(L_{+}^{n}+\alpha_{n} \sum_{i=1}^{N} q_{i} \partial^{-1} r_{i}\right) L-L\left(L_{+}^{n}+\alpha_{n} \sum_{i=1}^{N} q_{i} \partial^{-1} r_{i}\right) \\
& =\left[B_{n}+\alpha_{n} \sum_{i=1}^{N} q_{i} \partial^{-1} r_{i}, L\right] .
\end{aligned}
$$

Similarly, we can prove that $L$ satisfies (6b).

It is well known that the Wronskian determinant [4]

$$
W r\left(h_{1}, \ldots, h_{N}\right)=\left|\begin{array}{cccc}
h_{1} & h_{2} & \cdots & h_{N} \\
h_{1}^{\prime} & h_{2}^{\prime} & \cdots & h_{N}^{\prime} \\
\vdots & \vdots & \vdots & \vdots \\
h_{1}^{N-1} & h_{2}^{N-1} & \cdots & h_{N}^{N-1}
\end{array}\right|
$$

is a $\tau$-function of the $\mathrm{KPH}$ and the $N$ th order differential operator given by

$$
W=\frac{1}{W r\left(h_{1}, \ldots, h_{N}\right)}\left|\begin{array}{ccccc}
h_{1} & h_{2} & \cdots & h_{N} & 1 \\
h_{1}^{\prime} & h_{2}^{\prime} & \cdots & h_{N}^{\prime} & \partial \\
\vdots & \vdots & \vdots & \vdots & \\
h_{1}^{N} & h_{2}^{N} & \cdots & h_{N}^{N} & \partial^{N}
\end{array}\right|
$$


provides the dressing operator, where $h_{1}, h_{2}, \ldots, h_{N}$ are $N$ independent functions and satisfy $W\left(h_{i}\right)=0$.

This dressing operator $W$ is constructed as follows: Let $f_{i}, g_{i}$ satisfy

$$
\begin{aligned}
& f_{i, \gamma_{n}}=\partial^{n}\left(f_{i}\right), \quad f_{i, \sigma_{k}}=\partial^{k}\left(f_{i}\right), \\
& g_{i, \gamma_{n}}=\partial^{n}\left(g_{i}\right), \quad g_{i, \sigma_{k}}=\partial^{k}\left(g_{i}\right), \quad i=1, \ldots, N,
\end{aligned}
$$

and let $h_{i}$ be the linear combination of $f_{i}$ and $g_{i}$

$$
h_{i}=f_{i}+F_{i}\left(\alpha_{n} \gamma_{n}+\beta_{k} \sigma_{k}\right) g_{i}, \quad i=1, \ldots, N,
$$

with $F_{i}(X)$ being a differentiable function of $X, X=\alpha_{n} \gamma_{n}+\beta_{k} \sigma_{k}$.

Define

$$
q_{i}=-\dot{F}_{i} W\left(g_{i}\right), \quad r_{i}=(-1)^{N-i} \frac{W r\left(h_{1}, \ldots, \hat{h}_{i}, \ldots, h_{N}\right)}{W r\left(h_{1}, \ldots, h_{N}\right)}, \quad i=1, \ldots, N,
$$

where the hat ${ }^{\wedge}$ means rule out this term from the Wronskian determinant, $\dot{F}_{i}=\frac{d \alpha_{i}}{d X}$. We have the following theorem.

Theorem 3. Let $W$ be defined by (26) and (28), $L=W \partial W^{-1}, q_{i}$ and $r_{i}$ be given by (29), then $W, L, q_{i}, r_{i}$ satisfy $(25)$ and $\left(\gamma_{n}, \sigma_{k}\right)-K P H(6)$ and $(12)$.

To prove Theorem 3, we need several lemmas. The first one is given by Oevel and Strampp [20].

Lemma 1. $W^{-1}=\sum_{i=1}^{N} h_{i} \partial^{-1} r_{i}$.

Lemma 2 ([13]). The operator $\partial^{-1} r_{i} W$ is a non-negative differential operator and

$$
\left(\partial^{-1} r_{i} W\right)\left(h_{j}\right)=\delta_{i j}, \quad 1 \leq i, j \leq N .
$$

Proof of Theorem 3. For (25a), taking $\partial_{\gamma_{n}}$ to the identity $W\left(h_{i}\right)=0$, using $(27),(28)$, the definition (29) and Lemma 2, we find

$$
\begin{aligned}
0 & =\left(W_{\gamma_{n}}\right)\left(h_{i}\right)+\left(W \partial^{n}\right)\left(f_{i}\right)+\alpha_{n} \dot{F}_{i} W\left(g_{i}\right)+F_{i}\left(W \partial^{n}\right)\left(g_{i}\right) \\
& =\left(W_{\gamma_{n}}\right)\left(h_{i}\right)+\left(W \partial^{n}\right)\left(h_{i}\right)-\alpha_{n} q_{i} \\
& =\left(W_{\gamma_{n}}\right)\left(h_{i}\right)+\left(L^{n} W\right)\left(h_{i}\right)-\alpha_{n} \sum_{j=1}^{N} q_{j} \delta_{j i} \\
& =\left(W_{\gamma_{n}}+L_{-}^{n} W-\alpha_{n} \sum_{j=1}^{N} q_{j} \partial^{-1} r_{j} W\right)\left(h_{i}\right) .
\end{aligned}
$$

Since the non-negative difference operator acting on $h_{i}$ in the last expression has degree $<$ $N$, it cannot annihilate $N$ independent functions unless the operator itself vanishes. Hence (25a) is proved. Similarly, we can prove (25b). Then Proposition 1 leads to (6a) and $(6 \mathrm{~b})$. 
The first equation in (6c) is easy to be verified by a direct calculation, so it remains to prove the second equation in $(6 \mathrm{c})$. First, we see that

$$
\begin{aligned}
\left(W^{-1}\right)_{\gamma_{n}} & =-W^{-1} W_{\gamma_{n}} W^{-1}=-W^{-1}\left(L_{+}^{n}-L^{n}+\alpha_{n} \sum_{j=1}^{N} q_{j} \partial^{-1} r_{j}\right) \\
& =\partial^{n} W^{-1}-W^{-1} B_{n}-\alpha_{n} W^{-1} \sum_{j=1}^{N} q_{j} \partial^{-1} r_{j} \\
\left(W^{-1}\right)_{\sigma_{k}} & =\partial^{k} W^{-1}-W^{-1} B_{k}-\beta_{k} W^{-1} \sum_{j=1}^{N} q_{j} \partial^{-1} r_{j}
\end{aligned}
$$

On the other hand, from $W^{-1}=\sum h_{i} \partial^{-1} r_{i}$ we have

$$
\begin{aligned}
& \left(W^{-1}\right)_{\gamma_{n}}=\sum \partial^{n}\left(h_{i}\right) \partial^{-1} r_{i}+\sum h_{i} \partial^{-1} r_{i, \gamma_{n}}, \\
& \left(W^{-1}\right)_{\sigma_{k}}=\sum \partial^{k}\left(h_{i}\right) \partial^{-1} r_{i}+\sum h_{i} \partial^{-1} r_{i, \sigma_{k}} .
\end{aligned}
$$

It is obviously that $\alpha_{n}(32)-\beta_{k}(31)=\alpha_{n}(34)-\beta_{k}(33)$, i.e.

$$
\begin{aligned}
-\beta_{k} \sum & \partial^{n}\left(h_{i}\right) \partial^{-1} r_{i}-\beta_{k} \sum h_{i} \partial^{-1} r_{i, \gamma_{n}}+\alpha_{n} \sum \partial^{k}\left(h_{i}\right) \partial^{-1} r_{i} \\
& +\alpha_{n} \sum h_{i} \partial^{-1} r_{i, \sigma_{k}} \\
= & -\beta_{k}\left(\partial^{n} W^{-1}-W^{-1} B_{n}\right)_{-}+\alpha_{n}\left(\partial^{k} W^{-1}-W^{-1} B_{k}\right)_{-} \\
= & -\beta_{k} \sum \partial^{n}\left(h_{i}\right) \partial^{-1} r_{i}+\beta_{k} \sum h_{i} \partial^{-1} B_{n}^{*}\left(r_{i}\right)+\alpha_{n} \sum \partial^{k}\left(h_{i}\right) \partial^{-1} r_{i} \\
& -\alpha_{n} \sum h_{i} \partial^{-1} B_{k}^{*}\left(r_{i}\right) .
\end{aligned}
$$

The above equations gives

$$
\beta_{k} \sum h_{i} \partial^{-1}\left(r_{i, \gamma_{n}}+B_{n}^{*}\left(r_{i}\right)\right)-\alpha_{n} \sum h_{i} \partial^{-1}\left(r_{i, \sigma_{k}}+B_{k}^{*}\left(r_{i}\right)\right)=0,
$$

which implies the second equation in (6c) holds.

\section{N-Soliton Solutions for $\left(\gamma_{n}, \sigma_{k}\right)-\mathrm{KPH}$}

Using Theorem 3 , we can find $N$-soliton solutions to every equations in the $\left(\gamma_{n}, \sigma_{k}\right)$-KPH (6) and (12). Let us illustrate it by solving (15). We take the solution of (27) as follows

$$
\begin{gathered}
f_{i}:=\exp \left(\lambda_{i} x+\lambda_{i}^{2} y+\lambda_{i}^{3} t\right)=e^{\xi_{i}}, \quad g_{i}:=\exp \left(\mu_{i} x+\mu_{i}^{2} y+\mu_{i}^{3} t\right)=e^{\eta_{i}}, \\
h_{i}:=f_{i}+F_{i}\left(\alpha_{2} y+\beta_{3} t\right) g_{i}=2 \sqrt{F_{i}} \exp \left(\frac{\xi_{i}+\eta_{i}}{2}\right) \cosh \left(\Omega_{i}\right), \quad \Omega_{i}=\frac{1}{2}\left(\xi_{i}-\eta_{i}-\ln F_{i}\right) .
\end{gathered}
$$

For example, when $N=1, W=\partial-\frac{h^{\prime}}{h}$,

$$
L=W \partial^{-1} W=\partial+\frac{\left(\lambda_{1}-\mu_{1}\right)^{2}}{4} \operatorname{sech}^{2} \Omega_{1} \partial^{-1}+\cdots .
$$


The one-soliton solution for (15) with $N=1$ as follows

$$
\begin{aligned}
u & =\frac{\left(\lambda_{1}-\mu_{1}\right)^{2}}{4} \operatorname{sech}^{2} \Omega_{1}, \\
q_{1} & =\sqrt{\alpha_{2} F_{1 y}+\beta_{3} F_{1 t}}\left(\lambda_{1}-\mu_{1}\right) e^{\xi_{1}+\eta_{1}} \operatorname{sech} \Omega_{1}, \\
r_{1} & =\frac{1}{\sqrt{F_{1}}} e^{-\left(\xi_{1}+\eta_{1}\right)} \operatorname{sech} \Omega_{1} .
\end{aligned}
$$

In the case of $N=2$, the two-soliton solution for (15) is given

$$
\begin{aligned}
& u=\partial^{2} \ln \Theta, \\
& q_{1}=\left(\alpha_{2} F_{1 y}+\beta_{3} F_{1 t}\right) \frac{\left(\lambda_{1}-\mu_{1}\right)\left(\lambda_{2}-\mu_{1}\right)}{\Theta}\left(1+F_{2} \frac{\left(\lambda_{1}-\mu_{2}\right)\left(\mu_{2}-\mu_{1}\right)}{\left(\lambda_{1}-\lambda_{2}\right)\left(\lambda_{2}-\mu_{1}\right)} e^{\eta_{2}-\xi_{1}}\right) e^{\eta_{1}}, \\
& q_{2}=\left(\alpha_{2} F_{2 y}+\beta_{3} F_{2 t}\right) \frac{\left(\lambda_{2}-\mu_{2}\right)\left(\lambda_{1}-\mu_{2}\right)}{\Theta}\left(1+F_{1} \frac{\left(\lambda_{2}-\mu_{1}\right)\left(\mu_{1}-\mu_{2}\right)}{\left(\lambda_{2}-\lambda_{1}\right)\left(\lambda_{1}-\mu_{2}\right)} e^{\eta_{1}-\xi_{2}}\right) e^{\eta_{2}}, \\
& r_{1}=\frac{1+F_{2} e^{\eta_{2}-\xi_{2}}}{\lambda_{2}-\mu_{1}} e^{-\xi_{1}}, \quad r_{2}=\frac{1+F_{1} e^{\eta_{1}-\xi_{1}}}{\lambda_{2}-\mu_{1}} e^{-\xi_{2}},
\end{aligned}
$$

where

$$
\Theta=1+F_{1} \frac{\lambda_{2}-\mu_{1}}{\lambda_{2}-\lambda_{1}} e^{\eta_{1}-\xi_{1}}+F_{2} \frac{\mu_{2}-\lambda_{1}}{\lambda_{2}-\lambda_{1}} e^{\eta_{2}-\xi_{2}}+F_{1} F_{2} \frac{\mu_{2}-\mu_{1}}{\lambda_{2}-\lambda_{1}} e^{\eta_{1}+\eta_{2}-\xi_{1}-\xi_{2}}
$$

\section{Conclusion}

In contrast to the multi-component generalization of KPH, we generalize KPH by introducing new time series $\gamma_{n}$ and $\sigma_{k}$ and adding eigenfunctions as components. The $\left(\gamma_{n}, \sigma_{k}\right)$-KPH includes KPH and exKPH, and contains first type and second type as well as mixed type of KPESCS as special cases. The constrained flows of $\left(\gamma_{n}, \sigma_{k}\right)-\mathrm{KPH}$ can be regarded as the generalized GDH. We develop the dressing method for solving the $\left(\gamma_{n}, \sigma_{k}\right)$-KPH and present its $N$-soliton solutions.

\section{Acknowledgments}

This work was supported by National Basic Research Program of China (973 Program) (2007CB814800), National Natural Science Foundation of China (10901090, 11171175 and 11201477) and Chinese Universities Scientific Fund (2011JS041). The second author is Supported by Spectical Funds for Co-construction Project of Beijing.

\section{References}

[1] Y. Cheng, J. Math. Phys. 33 (1992) 3774.

[2] E. Date, M. Jimbo, M. Kashiwara and T. Miwa, J. Phys. Soc. Jpn. 50 (1981) 3806.

[3] E. Date, M. Jimbo, M. Kashiwara and T. Miwa, Publ. Res. Inst. Math. Sci. 18 (1982) 1077.

[4] L. A. Dickey, Soliton Equations and Hamiltonian Systems, 2nd edn. (World Scientific Publishing, River Edge, NJ, 2003).

[5] X. B. Hu and H. Y. Wang, Inverse Problem 22 (2006) 1903.

[6] X. B. Hu and H. Y. Wang, Inverse Problem 23 (2007) 1433. 
[7] M. Jimbo and T. Miwa, Publ. Res. Inst. Math. 19 (1983) 943.

[8] V. G. Kac and J. W. Van de Leur, J. Math. Phys. 44 (2003) 3245.

[9] B. Konopelchenko, J. Sidorenko and W. Strampp, Phys. Lett. A 157 (1991) 17.

[10] R. L. Lin, X. J. Liu and Y. B. Zeng, J. Nonlinear Math. Phys. 15 (2008) 333.

[11] R. L. Lin, Y. B. Zeng and W. X. Ma, Physica A 291 (2001) 287.

[12] X. J. Liu, R. L. Lin, B. Jin and Y. B. Zeng, J. Math. Phys. 50 (2009) 053506.

[13] X. J. Liu, Y. B. Zeng and R. L. Lin, Phys. Lett. A 372 (2008) 3819.

[14] W. X. Ma, Commun. Nonlinear Sci. Numer. Simul. 16 (2011) 722.

[15] V. K. Mel'nikov, Lett. Math. Phys. 7 (1983) 129.

[16] V. K. Mel'nikov, Comm. Math. Phys. 112 (1987) 639.

[17] V. K. Mel'nikov, Phys. Lett. A 128 (1988) 488.

[18] V. K. Mel'nikov, Phys. Lett. A 128 (1988) 488.

[19] E. Nissimov and S. Pacheva, Phys. Lett. A 244 (1998) 245.

[20] W. Oevel and W. Strampp, J. Math. Phys. 37 (1996) 6213.

[21] M. Sato and Y. Sato, Nonlinear Partial Differential Equations in Applied Science (NorthHolland, Amsterdam, 1983), pp. 259-271.

[22] J. W. Van de Leur, J. Math. Phys. 39 (1998) 2833.

[23] T. Xiao and Y. B. Zeng, J. Phys. A 37 (2004) 7143.

[24] Y. Q. Yao, X. J. Liu and Y. B. Zeng, J. Phys. A: Math. Theor. 42 (2009) 454026. 